\title{
Evaluación de las Virtudes y Fortalezas Humanas en Población de Habla Hispana
}

\author{
Alejandro C. Cosentino
}

\section{Resumen}

La Psicología Positiva es el estudio científico de las experiencias subjetivas positivas, los rasgos individuales positivos y las instituciones positivas. La comprensión de los rasgos individuales positivos consiste en el estudio de las virtudes y fortalezas del carácter. Luego de un prolongado hiato, nuevamente ha comenzado el estudio del carácter moral en la psicología científica. Se presenta el desarrollo de un instrumento para la evaluación de las virtudes y fortalezas humanas según la clasificación del carácter de 6 virtudes y 24 fortalezas de Peterson y Seligman [Peterson, C. \& Seligman, M.E.P. (2004). Character strengths and virtues: A handbook and classification. Washington, DC, EE.UU. American Psychological Association; Oxford University Press]. Análisis psicométricos preliminares del Inventario de Virtudes y Fortalezas (IVyF) sugieren que este inventario muestra una razonable confiabilidad y estructura factorial.

Palabras Clave: Fortalezas, Virtudes, Carácter

\begin{abstract}
Positive Psychology is the scientific study of the positive subjective experiences, positive individual traits, and positive institutions. Understanding positive individual traits consists of the study of the character strengths and virtues. After a long hiatus, the study of moral character has started up again in scientific psychology. The development of an assessment instrument of the character strengths and virtues based upon the Peterson and Seligman character
\end{abstract}

- Licenciado en Psicología. Universidad de Buenos Aires. E-mail: alejandrocosentino@yahoo.com.ar. 
classification of 6 virtues and 24 strengths is presented [Peterson, C. \& Seligman, M.E.P. (2004). Character strengths and virtues: A handbook and classification. Washington, DC, US: American Psychological Association; Oxford University Press.]. Preliminary psychometric analyses of the Inventario de Virtudes y Fortalezas (IVyF) suggest this inventory demonstrates reasonable reliability and factor structure.

Key Words: Strengths, Virtues, Character 


\section{Introducción}

Se define a la Psicología Positiva como la ciencia de la experiencia subjetiva positiva, los rasgos individuales positivos, las instituciones y comunidades positivas (Gable \& Haidt, 2005; Seligman \& Csikszentmihalyi, 2000). Se puede considerar a la Psicología Positiva como compuesta por tres áreas: subjetiva, individual y grupal. El área subjetiva refiere al estudio de las experiencias subjetivas valoradas positivamente, como el bienestar y el placer; la individual se refiere a las virtudes y fortalezas del carácter moral, como el coraje y la sabiduría; y la grupal se refiere a los grupos humanos en relación con los aspectos positivos de los individuos, como las instituciones que favorecen el desarrollo de mejores ciudadanos (Seligman \& Csikszentmihalyi). De estas tres áreas, se ha propuesto que la individual, que se ocupa del estudio del carácter moral, tiene preeminencia sobre las otras dos (Park \& Peterson, 2009).

El estudio del carácter moral ha tenido un desarrollo sinuoso en la psicología científica. Ha sido un tema de interés para varias importantes figuras de la psicología en la primera mitad del siglo pasado como Edward L. Thorndike, quien intensamente prestó atención a las nociones de carácter, virtud y moralidad: consideró que el carácter y el intelecto estaban entre los más importantes temas de la psicología conductista, y llegó a proponer el desarrollo de un instrumento cuantitativo para evaluar la moralidad, que estuviese escalado de modo similar a las medidas de inteligencia. Sin embargo, los términos carácter y virtud llegaron a suprimirse del léxico psicológico hasta tal punto que para los oídos de los psicólogos contemporáneos encontrar estos términos en el discurso académico probablemente sea como toparse con una disonancia exasperante, destello de ecos antiguos con resonancias victorianas o puritanas. Como casi siempre sucede, no se ha llegado a esta situación por efecto del simple azar, sino por la deliberada exclusión del léxico de la psicología científica de los términos relativos al carácter moral (McCullough \& Snyder, 2000).

Se ha señalado a Gordon Allport, con su respaldo exclusivo al lenguaje de la personalidad para describir diferencias individuales, como a un teórico comprometido con la exclusión de los términos como carácter o virtud. Allport, quien enfocó su carrera al proyecto de construir y promover una ciencia de la personalidad, argumentó que el lenguaje del carácter necesitaba ser expurgado del léxico de la psicología científica por sus connotaciones morales. Figuras posteriores de la psicología de la personalidad seguirían por el mismo camino, dando razones contra la utilidad del léxico basado en las virtudes y el carácter (McCullough \& Snyder, 2000).

McCullough y Snyder (2000) señalan que el cambio del léxico de las virtudes y el carácter por el léxico de la psicología de la personalidad ha tenido sus costos: a) 
las virtudes tradicionales fueron apenas revisadas dentro de la psicología científica, teniendo como resultado un análisis superficial de las fuentes clásicas de fortalezas humanas, como la sabiduría, la prudencia, la esperanza, la gratitud, la humildad o el perdón; b) la psicología científica, en cierta medida, tampoco pudo arrojar luz sobre las conceptualizaciones de las personas comunes sobre el florecimiento, la prosperidad o el desarrollo humano, que no se limitan simplemente a no tener una enfermedad mental, sino que incluye el ser una buena persona; y finalmente, c) tal vez como la consecuencia más significativa, los psicólogos científicos han mostrado dificultades para recomendar soluciones basadas en las virtudes para las contrariedades de la vida, por lo que las clásicas fortalezas, como el autocontrol, la esperanza, el perdón y la gratitud, que las personas comunes con tanta frecuencia intentan utilizar para prevenir o remediar sus propios suplicios, como la falta de autodisciplina, la desesperación, la tendencia a la represalia violenta, y la envidia, no están siendo utilizados con frecuencia o de manera explícita en las intervenciones educativas o terapéuticas de la mayoría de los psicólogos profesionales. Pero este panorama comenzó a modificarse.

Desde el inicio de la Psicología Positiva a fines del siglo pasado, ha comenzado el reingreso de los términos relativos a las virtudes y fortalezas del carácter al campo de la psicología científica. Fue a inicios de este siglo que Peterson y Seligman (2004) dieron un fuerte impulso al estudio científico de las virtudes y fortalezas del carácter. Un aporte sustancial ha sido su desarrollo de una clasificación de 6 virtudes que incluía a 24 fortalezas del carácter, que se consideró un paso importante y necesario para el progreso del estudio científico de la excelencia moral. Se ha tenido la expectativa de que el uso de esta clasificación no sólo impulsase la utilización de un vocabulario común en la investigación científica de los rasgos positivos, sino que facilitase la comunicación intra e intergrupos de investigadores y clínicos, así como con el público en general.

El camino del desarrollo de la clasificación de Peterson y Seligman (2004) se ha iniciado con la investigación de las respuestas que han dado sobre el comportamiento moralmente bueno las tradiciones filosóficas y religiosas de evidente y duradero impacto en la civilización humana, como el confucianismo y taoísmo (de China), el budismo e hinduismo (del sur asiático), la filosofía ateniense, el judaísmo, el cristianismo y el islamismo (de Occidente). Como resultado, se halló que en estas tradiciones se repetían seis virtudes fundamentales: el coraje, la justicia, la humanidad, la templanza, la sabiduría y la trascendencia. De esta convergencia sugirió un fundamento no arbitrario para una clasificación de virtudes y fortalezas, evitando de este modo el sesgo histórico o cultural (Dahlsgaard, Peterson \& Seligman, 2005). La clasificación de Peterson y Seligman de las seis virtudes y sus correspondientes fortalezas del carácter se presentan en el Cuadro 1. 
Peterson y Seligman (2004) han señalado que el enfoque empleado para el estudio del carácter siguió el espíritu de la psicología de la personalidad. Coherentemente, han propuesto que, por definición, las virtudes son estables pero maleables, y supusieron que las condiciones contextuales y situacionales, tanto los entornos físicos como los sociales, podrían facilitar o perjudicar la aparición o desarrollo de las fortalezas y virtudes.

Según Peterson y Seligman (2004), las fortalezas del carácter son los ingredientes psicológicos (procesos o mecanismos) que definen a las virtudes. Por ejemplo, la sabiduría, en tanto virtud, se manifiesta a través de las fortalezas curiosidad, amor por el saber, apertura mental, creatividad y perspectiva, fortalezas que son similares entre sí porque involucran la adquisición y el uso del conocimiento, pero, se diferencian unas de otras.

Peterson y Seligman (2004) han desarrollado un cuestionario de autoinforme para la evaluación de las 6 virtudes y 24 fortalezas, el Values in Action Inventory of Strengths (VIA-IS), que consiste de 240 ítems. Se puede completar online libremente desde fines de 2001 y cuenta con una versión accesible online en idioma español. Este inventario usa una escala Likert de cinco puntos para medir cuán frecuente es que uno se perciba a sí mismo realizando conductas representativas de las fortalezas y virtudes del carácter. Todas las escalas del VIA-IS tienen alfas de Cronbach $>.70$ y correlaciones test-retest $>.70$. El VIA-IS tiene también una versión de papel y lápiz con la cual se han realizado algunas investigaciones. Paralelamente, puede obtenerse del sitio Web la International Personality Item Pool (IPIP) una escala de 213 ítems creada en base a la clasificación de Peterson y Seligman, de dominio público. Todas las escalas de fortalezas del IPIP-VIA cuentan con alfas $>.70$.

Debe señalarse que las correspondencias entre las 6 virtudes y las 24 fortalezas de la clasificación de Peterson y Seligman (2004) no han surgido como consecuencia de algún análisis estadístico realizado sobre los puntajes de las fortalezas o virtudes (v.g., un análisis factorial sobre las fortalezas), sino exclusivamente sobre bases teóricas. Por el contrario, tres análisis factoriales exploratorios calculados sobre los puntajes de las fortalezas del VIA (Macdonald, Bore \& Munro, 2008; Peterson \& Seligman; Peterson, Park, Pole, D’Andrea \& Seligman, 2008) han coincidido en la extracción de cinco factores luego de una rotación varimax. Aunque Macdonald et al. no han informado qué variables ponían en los factores, las otras dos investigaciones han mostrado algunas discrepancias entre sí en las variables que cargaban en los cinco factores. Por una parte, Peterson y Seligman han extraído los factores (las fortalezas se muestran entre paréntesis): a) fortalezas interpersonales (v.g., bondad, amor, liderazgo, ciudadanía, humor), b) fortalezas intelectuales (v.g., creatividad, curiosidad, amor por el saber, 
apreciación de la belleza), c) fortalezas de la restricción (imparcialidad, humildad, prudencia y clemencia), y d) fortalezas emocionales (v.g., valentía, autorregulación, vitalidad, esperanza), fortalezas teológicas (v.g., gratitud y espiritualidad). Por otra parte, Peterson et al. han informado la extracción de cinco factores con autovalores > 1.0: a) factor interpersonal (bondad, amor, liderazgo, ciudadanía, humor e inteligencia social), b) factor cognitivo (creatividad, curiosidad, amor por el saber, apreciación de la belleza), c) factor templanza (imparcialidad, humildad, prudencia, clemencia), d) factor fortaleza (valentía, autorregulación, integridad, apertura mental, persistencia, perspectiva), y e) factor trascendencia (gratitud, espiritualidad, vitalidad, esperanza).

A continuación se presenta las definiciones de las fortalezas humanas correspondientes con la clasificación del carácter de Peterson y Seligman (2004; Park, Peterson \& Seligman, 2004). La presentación de las definiciones de las fortalezas tiene un objetivo triple: por una parte, en razón de que Peterson y Seligman han propuesto que las fortalezas son los ingredientes psicológicos de las virtudes, se conjetura que comprendiendo a qué se refieren aquellas, se recorrerá un importante trayecto en la comprensión de estas; por otra parte, se busca acercar a los lectores de lengua española los términos y conceptos que se están usando en el estudio del carácter; y finalmente, se satisface la necesidad de definir explícitamente las variables utilizadas en este estudio.

\section{Definiciones de las Fortalezas Humanas}

Fortalezas de la Sabiduría y el Conocimiento: Creatividad, Curiosidad, Apertura Mental, Amor por el Saber, Perspectiva.

Creatividad. La fortaleza creatividad implica la producción de ideas o comportamientos claramente originales (novedosos, sorprendentes o inusuales) y adaptativos (que hagan una contribución positiva a la propia vida o a la vida de los demás). Incluye la creación artística, pero no se limita a esta.

Curiosidad. La fortaleza curiosidad representa el deseo intrínseco por experimentar y conocer. Implica el reconocimiento activo, la búsqueda y la regulación de la propia experiencia en respuesta a situaciones desafiantes. Al vivenciarse curiosidad, como estado de motivación emocional positivo, se inicia y se persiste en comportamientos dirigidos a un objetivo en respuesta a señales estimulantes.

Apertura mental. La fortaleza apertura mental es la voluntad de buscar activamente evidencias en contra de las creencias, planes o metas preferidas de uno y, al hallar esas evidencias, evaluarlas de un modo justo o imparcial, siendo capaz de cambiar el punto de vista propio. 
Amor por el saber. La fortaleza amor por el saber describe, por una parte, el modo en que una persona se relaciona con habilidades e informaciones nuevas y, por la otra, el interés individual sólidamente establecido por el cual una persona se relaciona con contenidos específicos. Las personas con amor por el saber son cognitivamente activas y típicamente experimentan sentimientos positivos en el proceso de adquirir nuevas habilidades, satisfacer su curiosidad, incrementar sistemáticamente el conocimiento que poseen, y/o aprender algo completamente nuevo (de modo formal o autodidacta).

Perspectiva. La fortaleza del carácter perspectiva representa un nivel superior de conocimiento, juicio, y una capacidad para dar sabios consejos a los demás. Esta fortaleza le permite al individuo tratar preguntas difíciles e importantes sobre el desarrollo y significado de la vida. La perspectiva es útil para el bien o bienestar de uno mismo y de los demás.

\section{Fortalezas del Coraje: Valentía, Persistencia, Integridad, Vitalidad}

Valentía. La valentía es la disposición a actuar de forma voluntaria, quizá con miedo, en presencia de una circunstancia peligrosa, donde los riesgos importantes son evaluados razonablemente y sus consecuencias son aceptadas, en un esfuerzo por conseguir o perseverar en algún bien percibido para uno mismo o para los demás, reconociendo que ese bien podría no alcanzarse o concretarse. La acción debe estar orientada hacia fines superiores. Incluye la valentía física, pero no se limita a esta.

Persistencia. La persistencia se define como la continuación voluntaria de un comportamiento activo dirigido a una meta a pesar de los obstáculos, dificultades o desalientos a fin de terminar lo que uno inició, sintiendo placer en la tarea terminada.

Integridad. La fortaleza integridad se refiere a ser moralmente probo y coherente consigo mismo, o sea, con los valores que uno propugna (practicando lo que uno predica). La persona íntegra es realista consigo misma, representando de forma exacta (de modo privado y público) sus estados internos, intenciones y principios. Esta fortaleza implica aceptar y hacerse responsable por los sentimientos y conductas de uno, tal cual son, cosechando valiosos beneficios de ello. Implica la justificación pública de las propias convicciones morales, aún cuando no sean aceptadas por los demás. El individuo íntegro tiene sensibilidad hacia las necesidades de los demás, que se demuestra al ayudar a otros en necesidad.

Vitalidad. La fortaleza vitalidad describe un aspecto dinámico del bienestar marcado por la experiencia subjetiva de sentirse vivo y con energía, que está directa e interactivamente relacionada con factores somáticos y psicológicos. A 
nivel somático, la vitalidad se vincula con la salud física, la ausencia de fatiga y el buen funcionamiento corporal. A nivel psicológico, la vitalidad representa un estado emocional activo y positivo que refleja experiencias de voluntad, efectividad e integración de sí mismo tanto a nivel interpersonal como intrapersonal. Las personas con vitalidad viven la vida como si fuese una aventura.

\section{Fortalezas de la Humanidad: Amor, Bondad, Inteligencia Social}

Amor. La fortaleza amor representa una orientación emocional, comportamental y cognitiva hacia los demás que se presenta en tres formas prototípicas. Amor hacia quienes son fuentes primarias de nuestros cuidados, protección y afecto; el prototipo es el amor de los hijos hacia sus padres. Amor por los individuos que dependen de nosotros; el prototipo es el amor de los padres por sus hijos. Amor que involucra el deseo apasionado de contacto emocional, físico y sexual con un individuo a quien consideramos especial y que nos hace sentir especiales; el prototipo es el amor romántico. La persona con amor considera valiosas las relaciones estrechas con los demás.

Bondad. La bondad indica una orientación de uno mismo hacia el otro que tiene una tonalidad emocional o afectiva vinculada a la reivindicación una humanidad compartida con el otro, al que se lo considera digno de atención, ayuda y respeto por derecho propio (no por el deber de respetar a los demás, ni a causa de sernos útil en algún sentido), y que genera comportamientos de ayuda hacia los demás.

Inteligencia social. Esta fortaleza incluye la capacidad de razonamiento sobre información emocional interna o externa (concerniente principalmente a motivaciones o sentimientos) que se utiliza en las relaciones con otras personas. La persona con esta fortaleza tiene una comprensión y evaluación exacta de sí mismo y de los demás; sabe qué hacer para ajustarse a diferentes situaciones sociales.

\section{Fortalezas de la Justicia: Ciudadanía, Imparcialidad, Liderazgo}

Ciudadanía. La ciudadanía representa un sentimiento de identificación con, y un sentido de obligación para, el bien común, que abarca a uno mismo pero que se extiende más allá del interés por uno. Se manifiesta en la defensa del interés de todos por sobre el interés privado. El individuo con estas características es un buen miembro de grupo, un buen compañero, es leal a su grupo, tiene un fuerte sentido del deber, y hace la parte que le corresponde por el bien de su grupo. El individuo con ciudadanía tiene un espíritu emprendedor social, compromiso y participación ciudadanos, y un sentido de la responsabilidad hacia la comunidad. 
Imparcialidad. La fortaleza imparcialidad es el producto del juicio moral: el proceso por el cual el individuo determina lo que es moralmente correcto, lo moralmente incorrecto y lo moralmente proscrito. Los individuos con esta fortaleza tienen juicio ecuánime para todas las relaciones sociales, son hábiles en la lógica abstracta de los pactos equitativos, son sensibles a temas de injusticia social, incorporan la compasión y protección hacia los demás, y tienen comprensión relacional. Los individuos con imparcialidad les dan su oportunidad a todos, y no dejan que sus sentimientos afecten su toma de decisiones sobre los demás. Son ciudadanos responsables, amigos confiables, y, en general, personas con moral.

Liderazgo. Esta fortaleza es una cualidad personal que se refiere a una constelación integrada de atributos temperamentales y cognitivos orientados a influenciar y ayudar a los miembros del grupo de uno. El individuo con esta fortaleza aspira a ocupar roles dominantes, y dispone confortablemente en un sistema integrado sus propias actividades y las actividades de los demás. Motiva y conduce las acciones grupales hacia el éxito colectivo, a la vez que se mantienen las buenas relaciones entre los miembros del equipo.

Fortalezas de la Templanza: Clemencia y Misericordia, Humildad/Modestia, Prudencia, Autorregulación

Clemencia y misericordia. La fortaleza clemencia representa un conjunto de cambios prosociales que ocurren en un individuo ofendido o dañado por alguien cercano, en el que las motivaciones básicas o tendencias de acción hacia el trasgresor se tornan más positivas (v.g., benévolas, tolerantes o generosas) y menos negativas (v.g., vengativas o evitativas). La clemencia puede considerarse como una forma especializada de misericordia, que es un concepto más general que refleja benevolencia o compasión hacia un trasgresor, o alguien sobre quien uno posee autoridad, o alguien en una gran aflicción. La persona con esta fortaleza no es vengativa y da una segunda chance a los demás.

Humildad/Modestia. La humildad y la modestia refieren primariamente a la estimación precisa (no subestimación) de los logros, talentos o méritos de uno, dejando que los méritos hablen por sí mismos ante los demás. La humildad se extiende a otros temas, como la sobriedad en la conducta social y en la forma de vestir. Implica, relativamente hacer poco foco sobre el yo, o tener capacidad para olvidarse de uno mismo. El individuo humilde reconoce el valor de todo y de las diferentes formas en que personas y cosas pueden contribuir con nuestro mundo. La persona con esta fortaleza está abierta a consejos, ideas nuevas e información contradictoria. 
Prudencia. La fortaleza prudencia es una orientación cognitiva hacia el futuro personal, una forma de razonamiento práctico y de autogestión para alcanzar eficazmente los propios objetivos de largo plazo. Los individuos prudentes tienen visión de futuro y presentan una preocupación deliberada por las consecuencias de sus acciones y decisiones. Los individuos con prudencia resisten exitosamente sus impulsos de expresarse o de hacer ciertas elecciones que satisfagan metas a corto plazo a expensas de los objetivos de largo plazo. Poseen un enfoque flexible y moderado sobre la vida, y luchan para que se establezca un equilibrio entre las metas y los fines de uno.

Autorregulación. La fortaleza autorregulación refiere a regular, controlar o gobernar las respuestas propias (v.g., acciones, expresiones, impulsos, apetitos, pensamientos o emociones) a fin de adaptarse a un cierto patrón o cumplir ciertos objetivos (v.g., ideales, normas morales, normas en general, objetivos de rendimiento o expectativas de otras personas). No sería óptimo o adaptativo responder a cada estímulo interno y externo que constantemente se recibe.

Fortalezas de la Trascendencia: Apreciación de la Belleza y la Excelencia, Gratitud, Esperanza, Humor, Espiritualidad

Apreciación de la belleza y la excelencia. La apreciación de la belleza y la excelencia (o simplemente apreciación) se refiere a la capacidad de encontrar, reconocer y sentir sutiles emociones autotrascendentes como embelesamiento y emociones relacionadas (v.g., admiración, asombro o éxtasis) respecto a lo bueno del entorno físico (belleza) y social (talento y virtud moral): de la naturaleza, las artes, las matemáticas, las ciencias, las experiencias cotidianas, o de cualquier dominio en que pueda reconocerse excelencia.

Gratitud. La fortaleza gratitud es un sentimiento de agradecimiento y alegría que surge como respuesta a reconocer que uno se ha beneficiado, como cuando uno recibe un beneficio tangible por la acción de otra persona (como recibir un obsequio), o simplemente porque una persona específica existe. Esta fortaleza también refiere a un momento de alegría expansiva y pacífica evocada por la belleza natural. La persona con esta fortaleza expresa su agradecimiento.

Esperanza. La fortaleza esperanza representa una actitud cognitiva, emocional y motivacional hacia el futuro. El individuo con esta fortaleza piensa en el futuro con la expectativa positiva de que se van a producir los acontecimientos y los resultados deseados, actuando de una manera que supone que los hace más probables de que sucedan, y sintiéndose confiado de que, con los esfuerzos apropiados, va a conseguir mantener el buen humor en el aquí y ahora e impulsar acciones dirigidas a la meta. 
Humor. El humor implica el reconocimiento lúdico, el disfrute risueño, y/o la creación de incongruencias. El individuo con esta fortaleza tiene una visión alegre y serena que le permite ver el lado positivo de las cosas ante la adversidad y, en consecuencia, mantener un buen estado de ánimo. La persona con humor hace bromas, y hace reír o sonreír a los demás.

Espiritualidad. La espiritualidad se refiere a creencias y prácticas que se basan en la convicción de que hay una dimensión trascendente (no física) de la vida. El individuo con espiritualidad cree en propósitos y significados superiores, y sabe dónde encaja en un esquema superior de la vida. Estas creencias proveen sustento emocional y dan forma a sus conductas.

\section{Desarrollo de un Inventario para la Evaluación de las Fortalezas Humanas}

El desarrollo de un inventario para evaluar las fortalezas y virtudes ha sido producto de desafíos prácticos. Se había planeado llevar a cabo una investigación sobre el carácter en cadetes del ejército argentino, por lo que se necesitaba contar con un instrumento en castellano, adaptado a la cultura argentina, para la evaluación del carácter. Antes del inicio del proyecto, no había ningún inventario disponible. En consecuencia, se consideró la posibilidad de realizar una adaptación del VIA-IS en inglés o bien utilizar una adaptación de la versión española. Sin embargo, la longitud del cuestionario, de 240 ítems, y el tiempo en completarlo, de una hora aproximadamente en población militar (Matthews, Eid, Kelly, Bailey \& Peterson, 2006), no se ajustaba a los requerimientos para la realización de la investigación de administrar cuestionarios de pocos ítems y rápidos de completar. Para ajustar la investigación a esos límites, se tomó la decisión de desarrollar un cuestionario para evaluar fortalezas con la mínima cantidad de ítems y utilizando autodescripciones de un párrafo de longitud.

Se consultó las definiciones consensuales de cada fortaleza de Peterson y Seligman (2004) y los inventarios VIA-IS de los mismos autores, tanto la versión online como la versión en papel y lápiz, a fin de que cada autodescripción del inventario contase con una validez adecuada. Como paso siguiente, se tradujeron y adaptaron al español los ítems del IPIP-VIA, que se consultaron para crear 24 párrafos diferentes que representaban la descripción de sí misma que hacía una persona que poseía una fortaleza del carácter específica (autodescripción positiva). Se intentó que los párrafos fuesen gramatical y léxicamente simples. Luego se desarrolló, para cada ítem, una autodescripción opuesta, de ausencia de la fortaleza, a fin de producir ítems bipolares. La autodescripción negativa era un párrafo que negaba, ordenada y respectivamente, cada una de las declaraciones de la autodescripción positiva, intentando mantener la estructura gramatical y las 
palabras que estaban en la autodescripción con presencia de la fortaleza. Se le dio formato a los 24 ítems de modo que la mitad de los ítems bipolares presentase a la izquierda la autodescripción negativa, que constituía un ítem de puntuación directa. Luego, se ordenaron los 24 ítems bipolares de modo de equilibrar la sucesión de los ítems de puntuación directa e inversa. Finalmente, se anexó a cada ítem una escala tipo Likert de 5 opciones de respuesta, de modo que el encuestado pudiese indicar a quién se parecía más, si a una persona con presencia o a una persona con ausencia de la fortaleza.

El inventario producido se administró de modo piloto a una muestra de 48 cadetes de tercer año del ejército. En general, demoraron menos de media hora en completarlo y pareció ser comprendido adecuadamente. Debe tenerse presente que la población militar está constituida por personas provenientes de diferentes puntos de la geografía Argentina. Posteriormente, el inventario se administró a dos grupos independientes de estudiantes universitarios, con resultados similares. A la versión definitiva del inventario se la llamó Inventario de Virtudes y Fortalezas.

\section{Inventario de Virtudes y Fortalezas (IVyF)}

El IVyF es un inventario desarrollado para evaluar las 6 virtudes y 24 fortalezas del carácter según la clasificación de Peterson y Seligman (2004), construido por Alejandro C. Cosentino y Alejandro Castro Solano en 2008 (manuscrito en preparación). Este inventario de papel y lápiz, incluye 24 ítems bipolares con 5 opciones de respuesta tipo Likert, para que los encuestados indiquen en qué grado se parecen más a dos autodescripciones enfrentadas, una de ausencia de la fortaleza y la otra de presencia. La puntuación de cada ítem va de 1 (Soy muy parecido a la $1^{a}$ persona) a 5 (Soy muy parecido a la $2^{a}$ persona), correspondiendo el puntaje mayor, a mayor presencia de la fortaleza. La mitad de los ítems se puntúan de forma directa. Los puntajes de las escalas de virtudes se obtienen promediando el puntaje de los ítems que corresponden a cada virtud.

Se presenta como ejemplo al ítem bipolar que evalúa la fortaleza persistencia, constituido por dos autodescripciones opuestas: a) la autodescripción de presencia de la fortaleza es Cuando trabajo, no me distraigo ni dejo mis tareas sin terminar. Y como no soy de abandonar enseguida, si aparece un obstáculo tiendo a seguir haciendo lo que había decidido hacer hasta terminarlo. Así que, no hay cosas que empiece que deje sin terminar. Además, me pongo metas en la vida, y b) la autodescripción de ausencia de la fortaleza es Cuando trabajo, me distraigo y dejo mis tareas sin terminar. Y como soy de abandonar enseguida, si aparece un obstáculo no tiendo a seguir haciendo lo que había decidido hacer hasta terminarlo. Así que, hay cosas que empiezo y que dejo sin terminar. Además, no me pongo metas en la vida. 
Cuadro 1 | Clasificación de las virtudes y fortalezas del carácter según Peterson y Seligman

\begin{tabular}{ll}
\hline Virtud & Fortaleza \\
\hline Coraje & Valentía, persistencia, integridad, vitalidad. \\
\hline Humanidad & Amor, bondad, inteligencia social. \\
\hline Justicia & Ciudadanía, imparcialidad, liderazgo. \\
\hline Sabiduría y conocimiento & $\begin{array}{l}\text { Creatividad, curiosidad, apertura mental, amor por } \\
\text { el saber, perspectiva. }\end{array}$ \\
\hline Templanza & Clemencia y misericordia, humildad/modestia, \\
& prudencia, autorregulación. \\
\hline Trascendencia & Apreciación de la belleza y la excelencia, gratitud, \\
& esperanza, humor, espiritualidad. \\
\hline
\end{tabular}

\section{Análisis Psicométrico Preliminar del IVyF}

\section{Método}

\section{Participantes y Procedimiento}

La muestra estuvo constituida por 781 personas de población general (453 mujeres y 328 varones). La media de edad fue de 40.9 años $(D T=16.7)$. Los voluntarios no recibieron retribución por su participación. Con esta muestra se evaluó la estabilidad y la validez factorial de los puntajes del IVyF. Una submuestra de 123 personas (70 mujeres y 53 varones; $M=37.8$ años, $D T=15.6$ ) recibió una segunda administración del IVyF a fin de evaluar la estabilidad del inventario.

\section{Análisis de Datos}

Por una parte, se realizaron cálculos de confiabilidad: se empleó alfa de Cronbach para evaluar la consistencia interna de los puntajes del IVyF, y se utilizó el método test-retest por medio del cálculo del coeficiente $r$ sobre los puntajes obtenidos de las dos administraciones del inventario separadas aproximadamente 3 semanas entre sí a fin de evaluar la estabilidad. Por otra parte, se realizó un análisis factorial exploratorio con el método de componentes principales con el objetivo de identificar factores que expliquen la configuración de las correlaciones de los ítems. La extracción de factores se basó en el método de los autovalores > 1.0. Se utilizó el método de rotación varimax. Los análisis de los datos se realizaron con el programa PASW Statistics 17. Todos los valores informados de $p$ son bilaterales. 


\section{Resultados}

\section{Confiabilidad}

Consistencia interna. En el Cuadro 2, se informa los coeficientes alfa de Cronbach, que evalúan la consistencia interna para los puntajes de las subescalas de virtudes. El alfa de Cronbach es una medida de confiabilidad del tipo consistencia interna que debe interpretarse con el número de ítems en mente, debido a que el coeficiente aumenta a medida que la cantidad de ítems aumenta (Cortina, 1993). Para pocos ítems, no es esperable hallar un valor muy elevado del alfa de Cronbach. Finalmente, con el objetivo de evaluar la consistencia interna de los puntajes de la totalidad del IVyF, se calcularon dos alfa de Cronbach (Cuadro 2): el primero sobre los puntajes de los 24 ítems individuales - i.e., las fortalezas(Macdonald et al., 2008), que se denomina escala total, y el otro sobre los puntajes de las 6 subescalas - i.e., las virtudes-, que se denomina escala compuesta (Helms, Henze, Sass \& Mifsud, 2006).

Estabilidad. Se informa en el Cuadro 2 las confiabilidades de tipo estabilidad para las virtudes y fortalezas obtenidas a través de la técnica test-retest. Las correlaciones test-retest son particularmente importantes para apreciar la confiabilidad de la medición de las fortalezas, ya que no se calcularon índices de consistencia interna de las fortalezas porque se miden a través de un sólo ítem (Gosling, Rentfrow \& Swann, 2003). Además, para mostrar la estabilidad del puntaje general del IVyF, se muestra las correlaciones test-retest de los puntajes de la escala total y de la escala compuesta. 


\section{Cuadro 2 | Confiabilidades sobre los puntajes del IVyF}

\begin{tabular}{|c|c|c|}
\hline Variable & Estabilidad $^{a}$ & Congruencia interna $^{b}$ \\
\hline \multicolumn{3}{|c|}{ FORTALEZAS } \\
\hline Persistencia & .82 & - \\
\hline Valentía & .78 & - \\
\hline Integridad & .74 & - \\
\hline Vitalidad & .79 & - \\
\hline Amor & .76 & - \\
\hline Inteligencia social & .82 & - \\
\hline Bondad & .81 & - \\
\hline Imparcialidad & .72 & - \\
\hline Liderazgo & .87 & - \\
\hline Ciudadanía & .73 & - \\
\hline Creatividad & .85 & - \\
\hline Apertura mental & .79 & - \\
\hline Curiosidad & .82 & - \\
\hline Perspectiva & .80 & - \\
\hline Amor por el saber & .79 & - \\
\hline Autorregulación & .77 & - \\
\hline Clemencia y misericordia & .79 & - \\
\hline Prudencia & .78 & - \\
\hline Humildad/Modestia & .87 & - \\
\hline Apreciación & .78 & - \\
\hline Gratitud & .84 & - \\
\hline Espiritualidad & .92 & - \\
\hline Esperanza & .75 & - \\
\hline Humor & .77 & - \\
\hline \multicolumn{3}{|c|}{ VIRTUDES } \\
\hline Coraje & .86 & .59 \\
\hline Humanidad & .87 & .55 \\
\hline Justicia & .83 & .50 \\
\hline Sabiduría y conocimiento & .89 & .63 \\
\hline Templanza & .87 & .49 \\
\hline Trascendencia & .92 & .57 \\
\hline \multicolumn{3}{|c|}{ OTROS PUNTAJES } \\
\hline IVyF (escala compuesta) & .93 & .81 \\
\hline IVyF (escala total) & .94 & .85 \\
\hline
\end{tabular}

Nota

Apreciación = Apreciación de la belleza y la excelencia. $\mathrm{IVyF}=$ Inventario de Virtudes y Fortalezas.

aTest-retest a través de $r$ sobre una muestra de población general, $n=123$. Todos los $r$ s con $p s<.001$, bilaterales. ${ }^{\mathrm{b}}$ Consistencia interna a través de alfa de Cronbach sobre muestra población general $(n=781)$. 


\section{Validez Factorial del IVyF}

Debido a que el valor de la medida de adecuación muestral de Kaiser-MeyerOlkin (KMO) fue de .89, y el valor de la prueba de esfericidad de Bartlett fue estadísticamente significativo $\left(x^{2}=4205.81, g l=276, p<.001\right)$, se concluyó que era apropiado efectuar un análisis factorial exploratorio sobre el IVyF. Se extrajeron 5 factores que se muestran en el Cuadro 3.

\section{Cuadro 3 | Matriz de factores rotados sobre los puntajes del IVyF de las 24 fortalezas del carácter}

\begin{tabular}{|c|c|c|c|c|c|}
\hline & Factor 1 & Factor 2 & Factor 3 & Factor 4 & Factor 5 \\
\hline Porcentaje de la varianza & 12.27 & 10.80 & 8.92 & 8.71 & 7.57 \\
\hline \multicolumn{6}{|l|}{ Variable } \\
\hline Humor & .63 & & & & \\
\hline Ciudadanía & .63 & & & & \\
\hline Liderazgo & .60 & & & & \\
\hline Amor & .60 & & & & \\
\hline Inteligencia Social & .58 & & & & \\
\hline Bondad & .50 & & & & \\
\hline Persistencia & & .68 & & & \\
\hline Autorregulación & & .58 & & & \\
\hline Vitalidad & & .56 & & & \\
\hline Valentía & & .51 & & & \\
\hline Esperanza & & .50 & & & \\
\hline Humildad/Modestia & & & .72 & & \\
\hline Prudencia & & & .65 & & \\
\hline Integridad & & & .49 & & \\
\hline Apertura mental & & & .48 & & \\
\hline Imparcialidad & .41 & & .46 & & \\
\hline Amor por el Saber & & & & .76 & \\
\hline Apreciación & & & & .59 & \\
\hline Creatividad & & & & .52 & \\
\hline Curiosidad & & & & .47 & \\
\hline Perspectiva & & & & .43 & \\
\hline Espiritualidad & & & & & .73 \\
\hline Clemencia y misericordia & & & & & .56 \\
\hline Gratitud & & & & & .47 \\
\hline
\end{tabular}

Nota. No se muestran los coeficientes menores a .40. Apreciación = Apreciación de la belleza y la excelencia. IVyF = Inventario de Virtudes y Fortalezas. 


\section{Comentarios Finales}

Cuatro análisis psicométricos calculados sobre los puntajes de las fortalezas del VIA son puntos de referencia para esta validación preliminar del IVyF (Macdonald et al., 2008; Peterson \& Seligman, 2004; Peterson et al., 2008; IPIP).

Las confiabilidades de los datos del IVyF son similares a los hallados por esas investigaciones. En relación a la validez factorial, hay que subrayar que los análisis factoriales sobre las fortalezas (Macdonald et al., 2008; Peterson \& Seligman, 2004; Peterson et al., 2008) han puesto en evidencia que las agrupaciones de fortalezas resultantes no coinciden fielmente con los racimos de fortalezas agrupados por virtud que han propuesto Peterson y Seligman. Recuérdese que su clasificación se generó exclusivamente sobre bases teóricas, y no como resultado de algún análisis estadístico complejo.

El resultado del análisis factorial exploratorio del IVyF es coincidente con los resultados de las tres investigaciones que realizaron este tipo de análisis: se han extraído cinco factores de los puntajes del IVyF, que luego se rotaron ortogonalmente. Aunque sólo Macdonald et al. (2008) presentaron el gráfico de sedimentación, su estructura resultó ser muy similar a la hallada en esta investigación. Especialmente, la composición de los factores del IVyF ha coincidido a la de los factores informados por Peterson y Seligman (2004) que corresponden a las fortalezas interpersonales, intelectuales, de la restricción, emocionales y teológicas.

Se concluye que el IVyF es una medida breve adecuada para la evaluación rápida de las fortalezas humanas según la clasificación del carácter de Peterson y Seligman (2004). Además de los análisis psicométricos preliminares presentados en esta investigación que han mostrado que los puntajes del IVyF muestran una adecuada confiabilidad y validez de constructo, se están desarrollando análisis complementarios de las propiedades psicométricas de este inventario.

Un comentario más general para finalizar. A pesar del entusiasmo que despierta los avances en la ciencia de las fortalezas humanas, Aspinwall y Staudinger (2003) han sostenido que tres riesgos potenciales deben tenerse presente. En primer lugar, y quizás el más importante, se debe evitar utilizar los resultados de la investigación sobre el carácter moral para prescribir. Podría haber un pequeño paso desde la investigación de las fortalezas humanas, a fin de mejorar el bienestar de las personas, a la adopción de un sistema determinado de valores y la prescripción de ese sistema. La segunda advertencia se refiere a la posibilidad de que haya situaciones y contextos donde los atributos o procesos que funcionan como fortalezas en un ambiente puedan funcionar como debilidades en otro, y viceversa. Por ejemplo, a pesar de los buenos resultados asociados al optimismo, se ha encontrado que el pesimismo es más adaptativo en algunas personas y en algunas 
culturas no occidentales. La tercera precaución proviene de la observación de que no todo lo que brilla es oro. Sería un grave error suponer que todo lo positivo es bueno, es decir, que todas las creencias, características y experiencias positivas tienen efectos favorables sobre el bienestar y la salud, tanto para las personas como para las redes sociales. Debe investigarse a fin de esclarecer en cuáles situaciones lo positivo está vinculado con resultados benignos y cuándo no lo está, a fin de adoptar una posición realista y equilibrada en la ciencia de las fortalezas humanas.

\section{Referencias Bibliográficas}

Aspinwall, L.G. \& Staudinger, U.M. (2003). A psychology of human strengths: Some central issues of an emerging field. En L.G. Aspinwall \& U.M. Staudinger (Eds.), A psychology of human strengths: Fundamental questions and future directions for a positive psychology. (pp. 9-22). Washington, DC, EE.UU. American Psychological Association.

Cortina, J.M. (1993). What is coefficient alpha? An examination of theory and applications. Journal of Applied Psychology, 78, 98-104.

Dahlsgaard, K., Peterson, C. \& Seligman, M.E.P. (2005). Shared virtue: The convergence of valued human strengths across culture and history. Review of General Psychology, 9, 203-213.

Gable, S.L. \& Haidt, J. (2005). What (and why) is positive Psychology? Review of General Psychology, 9, 103-110.

Gosling, S.D., Rentfrow, P.J. \& Swann Jr., W.B. (2003). A very brief measure of the Big-Five personality domains. Journal of Research in Personality, 37, 504-528.

Helms, J.E., Henze, K.T., Sass, T.L. \& Mifsud, V.A. (2006). Treating Cronbach’s alpha reliability coefficients as data in counseling research. The Counseling Psychologist, 34, 630-660.

International Personality Item Pool: A scientific collaboratory for the development of advanced measures of personality traits and other individual differences (http:// ipip.ori.org/). Sitio web.

Macdonald, C., Bore, M. \& Munro, D. (2008). Values in action scale and the Big 5: An empirical indication of structure. Journal of Research in Personality, 42, 787-799.

Matthews, M.D., Eid, J., Kelly, D., Bailey, J.K.S. \& Peterson, C. (2006). Character strengths and virtues of developing military leaders: An international comparison. Military Psychology, 18, S57-S68. 
McCullough, M.E. \& Snyder, C.R. (2000). Classical source of human strength: Revisiting an old home and building a new one. Journal of Social \& Clinical Psychology, 19, 1-10.

Park, N. \& Peterson, C. (2009). Strengths of character in schools. En R. Gilman, E.S. Huebner \& M.J. Furlong (Eds.), Handbook of Positive Psychology in schools (pp. 65-76). NY, EE.UU: Routledge.

Park, N., Peterson, C. \& Seligman, M.E.P. (2004). Strengths of character and well-being. Journal of Social \& Clinical Psychology, 23, 603-619.

Peterson, C. \& Seligman, M.E.P. (2004). Character strengths and virtues: A handbook and classification. Washington, DC, EE.UU: American Psychological Association; Oxford University Press.

Peterson, C., Park, N., Pole, N., D’Andrea, W. \& Seligman, M.E.P. (2008). Strengths of character and posttraumatic growth. Journal of Traumatic Stress, 21, 214-217.

Seligman, M.E.P. \& Csikszentmihalyi, M. (2000). Positive psychology: An introduction. American Psychologist, 55, 5-14.

\section{Nota Acerca del Autor}

Alejandro C. Cosentino, Facultad de Psicología, Universidad de Buenos Aires. El autor está actualmente desarrollando su tesis doctoral Virtudes y Fortalezas del Carácter: Su Relación con la Personalidad y el Rendimiento Académico bajo la dirección del Prof. Dr. Alejandro Castro Solano en la Facultad de Humanidades y Ciencias Sociales de la Universidad de Palermo.

El autor agradece profundamente a su director de tesis Prof. Dr. Alejandro Castro Solano por su valioso apoyo para el desarrollo de este manuscrito.

La correspondencia puede ser enviada a la dirección postal calle Humberto $1^{\circ}$ $24601^{\circ} \mathrm{G}$ (C1229AAN), Capital Federal, República Argentina. Teléfono: 11-43086426. 
